\title{
Germanica
}

GERMANICA $7 \mid 1990$

Grenze und Entgrenzung

\section{Die Schmerzen der auslaufenden Art}

Jurij Koch

\section{OpenEdition}

Journals

Édition électronique

URL : http://journals.openedition.org/germanica/2513

DOI : 10.4000/germanica.2513

ISSN : 2107-0784

\section{Éditeur}

Université de Lille

\section{Édition imprimée}

Date de publication : 30 juin 1990

Pagination : 157-164

ISSN : 0984-2632

\section{Référence électronique}

Jurij Koch, « Die Schmerzen der auslaufenden Art », Germanica [Online], 7| 1990, Online erschienen am: 18 Juli 2014, abgerufen am 06 Oktober 2020. URL : http://journals.openedition.org/germanica/ 2513 ; DOI : https://doi.org/10.4000/germanica.2513

Ce document a été généré automatiquement le 6 octobre 2020.

(c) Tous droits réservés 


\title{
Die Schmerzen der auslaufenden Art
}

\author{
Jurij Koch
}

1 Für das linke obere Viertel des mehrfach geknickten Stadt- und Landkreises Cottbus hat man sich an der Geographisch-Kartographischen Anstalt Hermann Haack in Gotha für Grün entscheiden können. In zwei unbedeutenden Abstufungen. Zwischen 40 und $80 \mathrm{~m}$ über dem Meeresspiegel.

2 Hier also. In diesem weichseleiszeitlichen Schwemmsandfacher. In dem sich nach Osten hin ausleckenden Spreewald. Bis nach Pietz. Fast bis nach Jänschwalde. Wo das neueste Kraftwerk steht. Aber die von ihm wegführenden Stromleitungen zeigt die Karte nicht. Nur das blauädrige Versorgungsnetz der Natur, die Fließe und Gräben, die Carna grobla, die Goramśica, die Małksa, die Patśowina und die Młynska Sprjewaja, die Hauptspree also. Alle fließen sie westwärts durch die noch sorbischen Dörfer Debsk und Majberg und Dešno und Strjažow und Prjawoz.

Hier, sagt J., der Ornithologe. Hier könnten wir sie sehn. Wenn wir Glück haben.

Und es gibt sie nur noch in zwei Exemplaren? frage ich.

5 In zwei oder drei, antwortet er. Nur hier. Sonst nirgends mehr im Land.

6 Der Vogel, von dem einschubweise die Rede sein wird, heißt Blauracke. Im Volksmund Mandelkrähe. Aber das Volk nimmt ihn weder so noch so in den Mund. Es kennt ihn nicht mehr. Das Interesse für ihn hat sich in die Ornithologie zurückgezogen. Auf ein paar Vogelgänger. Aber bei denen hält es sich. Und steigt mit dem Seltenheitswert, den zwei Exemplare einer Art und die nicht auszuschließende Gefahr ihres endlichen Ausbleibens bekommen können. Die Gefahr nämlich, daß die beiden Vögel auf ihr westlichstes europäisches Brutgebiet pfeifen, das Land südostwärts für immer verlassen, einfach von der winterlichen Reise aus Afrika nicht zurückkehrend. Schluß. Aus. Wieder eine Art weniger. Die Diversität gestutzt, die Vielfalt einfältiger. Man gewöhnt sich an das andauernde Ausbleiben, an den anthropogen bedingten Rückgang. Als wäre es menschheitlich belanglos, daß sich die Dinge der Natur umkehren. Die Entwicklungen gehen wieder vom höheren zum Niederen. Auffallen würde das Ausbleiben des bunten Vogels, des Coracias garrulus, zunächst allenfalls den Vogelkundlern, den Leuten mit dem sehr zeitgemäßen Vogel. Wie man überhaupt in einem Land wie dem meinen einen Vogel haben muß, um der normativischen Lethargie 
zu entkommen. Die wichtigsten Aufschlüsse über den Zustand unseres Daseins sind von Leuten mit einem Vogel zu erwarten, den «Verrückten», den ausgefallenen Typen. Nicht von Philosophen, die Rückgänge wie die an der Carna grobla und Goramsica entweder nicht zur Kenntnis nehmen würden, weil nicht sein kann, was nicht sein darf, oder sie pitzelten daraus einen Erfolg.

7 Ich bin vom Wahnsinn der Metapher besessen. Ich will wissen, ob die beiden Exemplare der Mandelkrähe noch im Land sind. Es liegt am Urinteresse meiner ethnischen Art, daß ich wissen muß, ob in meiner Zeit, vor meinen Augen (daß ich als Zeuge gerufen werden könnte, falls es zum Gerichtstag kommen sollte), ob in meiner Zeit vor meinen Augen etwas zu Ende gekommen ist, was nicht hätte zu Ende kommen sollen. Ich wünsche mir, der schöne Vogel möge noch dasein. So wie ich mir die Welt nur mit meiner ethnischen Art vorstellen kann. Sein, ihr Ausbleiben bedeuteten Verlust. Nach und nach wäre die Armut landesweit spürbar. Vielleicht sogar kontinental und planetar. Eine Farbe weniger. Zunahme der Grau werte. Ein Laut weniger, eine Sprache weniger. Zunahme der Schweigsamkeit. Es ist ein Irrtum - er könnte der Menscheit auf ihrer evolutionären Kehrtwendung kräftig helfen - es ist ein Irrtum zu glauben, die Völkerverständigung auf der Erde ginge schneller voran, wenn es weniger Sprachen gäbe, also sollte man sich um den Verlust einer winzigen, der kleinsten slawischen zumal, nicht soviel Gedanken machen. Anderes sei wichtiger, zum Beispiel, wie die Winter wohltemperiert und lichtklar zu überstehen sind, Pech, daß die Kohle, die man dazu braucht, gerade unter Dörfern liegt, in denen die Sprache noch gesprochen wird.

8 Aber ich warne wider alle rationalen Argumente vor der drohenden endgültigen Abfahrt des Vogels. Vor dem Versanden eines Ethnikums mitsamt seinen Farben im Kippenkies des kopfstehenden Tertiärs, selbst, wenn die fortschreitenden Assimilierungen nicht nur dem bergbaulichen Abraum, sondern anderen, gefährlicheren, weil ziemlich dummen freiwilligen Abräumungen geschuldet sind.

9 Mir sind die Rangordnungen nicht geheuer. Die Ordnungen, unter denen die Kohle immer, die Mandelkrähe niemals mit einem politischen Primat rechnen kann. Sie wird bei der Frage, was für uns im Augenblick wichtiger ist, immer ihren schönen Schwanz einziehen müssen. Das will mein fortschrittlich denkender Kopf nicht einsehen. Und ordnet Angst an: Wir könnten in unserer geheizten artenarmen Welt frieren. Ich beantrage ein Einspruchsrecht der Buntschwänzigen.

10 Es ist Mitte August. Wir machen uns auf zur ersten Fahrt ins biotopische Areal der Blauracke. An die Ufer der Carna grobla und Goramsica. Fast lächerlich die Vorstellung, wir könnten eins der beiden Exemplare vor die feldstechenden Augen bekommen. Mein Sohn Ilja glaubt jedenfalls an keinen guten Ausgang. Er bittet seinen Freund Silvio mitzukommen. Der stimmt unter der Bedingung zu, daß er auf den in Aussicht gestellten weiten Wiesen und Stoppelfeldern seine selbstgebauten Bumerang ausprobieren darf. Natürlich richten wir gleich hinter Peitz auf jeden Vogel unsere Gläser. Vor allem auf die, die «auf freien Warten und Telefondrähten» sitzen, auf vorbeifliegende Insekten wartend, ob nicht unter ihnen einer ist, der die Ausmaße von $30 \mathrm{~cm}$ überschreitet, mit «azurblauem Gefieder und kastanienbraunem Rücken, grünlichem Schwanz, lebhaft blauen Flügeln, die (im Fluge zu sehen) schwarz gesäumt sind». Nein, dermaßen aufsehenerregend präsentiert sich hier kein Vogel. Obwohl einer lange unsere auffallend vorsichtige Annäherung erträgt, allein auf dem durchhängenden Draht der Uberlandleitung sitzend. Die Jungs lenken mich für einen Augenblick ab. Im selben verläßt er seine industrielle Warte im Gleitflug durch die 
flimmernde Luft, abwärts dem Wäldchen zu, in dessen dunklen Färbungen er nicht mehr auszumachen ist. Der gesuchte war es nicht.

11 Hinter dem Wäldchen ist die Kiesgrube Garkoschka. Mit splitternackten Badenden am Strand. Wir hören nichts von ihnen. Auch die mächtigen Elektromaste, unter denen wir über ein Stoppelfeld laufen, stören nicht. Sie sind in ihrer statischen Ausgewogenheit, ihrer weitläufigen Nullperspektive, ihrer geometrischen Schönheit Bestandteil des Areals geworden. Angenommen von Vögeln und Bäumen. Auch der Bumerang, der sich unter ihnen ausprobieren läßt, wird keinen Vogel verjagen. Vom Kinderspiel geht die Störung nicht aus. Auch nicht von naturfreundlicher Technik, wie man sehen kann. Die Störung geht immer von machtbesessener Dummheit aus, von der Borniertheit des politischen Primats, von dem Protz, der sich immer im Geleitzug der menschlichen Kreativität befindet und die Vernunft versucht zu überholen. «Was einmal gedacht wurde, kann nicht zurückgenommen werden», sagt Dürrenmatts enttäuschter Physiker Möbius in der Komödie, in der einem das Lachen vergeht, in der der nützliche technische Geist in die Hände einer gemeingefährlichen buckligen Jungfer fällt. Hier, wo wir auf der Suche nach dem bunten Vogel sind, stört der Funkwagen der Landwirtschaftlichen Produktionsgenossenschaft, der auf der anderen Seite der Carna grobla steht, dessen Lautsprecher durch die links und rechts sperrangelweit geöffneten Türen des khakifarbenen LKW's Anweisungen des Dispatchers in die Landschaft plärren. Und ein mächtiger Traktor rattert, nein, sein achtzylindriger Motor PSt, daß die Kolbenstöße in den Wucherungen der beiden Ufer zu spüren sind. Auspuff qualm liegt zerfasert über dem Wasser des Fließes. Kompromißlerisch fortschrittsgläubig könnte man geneigt sein, in ihm den Rest des Morgennebels zu sehn. Dem Traktor voran fahrt ein Grashäcksler, zerschnipselnd den stattgrünen Wildwuchs. Warum, frage ich mich, fragen auch die Jungs, warum läßt man der Natur hier nicht ihre Ruhe? Ist der Einbruch ins Paradies der Mandelkrähe gerechtfertigt? Wodurch? Die Kalkulation bitte! Sind Gewinne und Verluste miteinander abgewogen? Ist überhaupt die Möglichkeit des Verlusts bedacht worden? Was hat hier die Erfindung des drahtlosen Meldewesens zu suchen, wenn nicht etwas zu ornithologischen Zwecken? Warum verläßt der khakifarbene LKW nicht den Archipel, die letzte Brut-und Heimstatt der Blauracke, die die sorbischen onomatopoetischen Namen Rjagawa und Rakajca trägt?

Wir brauchen Grenzen und das Verbot ihrer Überschreitung, wenn die Arbeit an einer grenzenlosen Welt ihren Sinn behalten soll.

Im 19. Jahrhundert lebte im Staate Washington das Volk der Duwamisch-Indianer. Der 14. Präsident der Vereinigten Staaten von Amerika, Franklin Pierce, wollte ihr Land für weiße Siedler kaufen. Seattle, der Häuptling der Duwamish, der sorbisch Sydler hieße, hielt im Jahre 1855 vor dem Präsidenten eine Rede. «Ich weiß nicht, unsere Art ist anders als die eure», soll er gesagt haben. «Wir wissen, daß der weiße Mann unsere Art nicht versteht... Er behandelt seine Mutter, die Erde, und seinen Bruder, den Himmel, wie Dinge zum Kaufen und Plündern... Das Tier, der Baum, der Mensch - sie alle teilen denselben Atem». Und weiter sprach der bunte Vogel Seattle: «Die Erde gehört nicht den Menschen, der Mensch gehört zur Erde... Der Mensch schuf nicht das Gewebe des Lebens, er ist darin nur eine Faser. Was immer ihr dem Gewebe antut, das tut ihr euch selber an... Wie kann ein Mensch seine Mutter besitzen?... Wenn die Büffel alle geschlachtet sind, die wilden Pferde gezähmt, die heimlichen Winkel des Waldes schwer vom Geruch vieler Menschen und der Anblick reifer Hügel geschändet von 
redenden Drähten - wo ist das Dickicht - fort, wo ist der Adler - fort... Das ist der Beginn bloßen Uberlebens».

So Seattle, Ursiedler Sydler, Häuptling Rakajca von der Carna grobla.

Wir leben in Umbruchzeiten. Den wohl wichtigsten der Menschheitsgeschichte. Am Ende Aufstiegseuphorie. Am Beginn des umfassendsten Zweifels. Der Umwertung der Werte. Es ist die große Zeit der Erhaltungen gekommen, der vielfaltigsten Bewahrungen: der Bewahrung des Friedens, des Lebens, der Welt, der Umwelt, ihrer schon übersehbaren Äußerungen. Das ist die Zeit der universalen Angst, daß sich die Evolution zu ihrem Ausgangspunkt zurückdrehen könnte, bis zum Urknall. Die des radikalsten Umdenkens also, daß den Philosophien vor Verblüffung der Atem stockt. Ihre bisherigen Überlegungen, wie sich die Menschen auf Erden am besten einrichten könnten, von Piaton über Campanella, Thomas Morus, Augustin, Saint Simon, Bakunin bis Marx endeten in der Vorstellung: Omnia sint communia, alles sei gemeinsam. Die Träumereien dieser sozialen Weltverbesserer, wie sie Ernst Bloch nennt, fanden ein gutes Ende mit dem Aufwachen im Morgengrauen, als der Schuß der Aurora auf der Newa fiel. Nun aber ist es an der Zeit, daß die Träumereien der Artenbewahrer beginnen: Omnia, quae sunt, maneant, alles, was ist, bleibe! Das gemeinsame Alles darf nicht in vernachlässigter Obhut der Allgemeinheit langsam aber sicher zum Wenig reduziert, am Ende der Vorstellung zum Nichts degeneriert werden. Dann hätte die Omniasintcommunia-Gesellschaft gerecht an alle nur noch Nichts zu verteilen. Alsdann ein infernalisches Gelächter aller über alle Philosophien, einschließlich des Sozialismus, ausbrechen könnte. Dann hätte wirklich alles nichts bedeutet.

Die Zeit der Bewahrungen ist zugleich die der Gebliebenen, der Kleinen, der Restlichen, der auf wenige Exemplare Reduzierten, der Gruppen, der Minoritäten, sozialen, biologischen, ethnischen. Sie, dessen exemplarisches Nochvorhandensein sowohl Glück als auch Gefahr anzeigen, besitzen eine besondere Sensibilität für alles, was Störung bedeuten könnte. Sie, wir, die Nochvorhandenen, die wenigen Bunt schwänzigen, wissen, fühlen, ahnen die Verarmung, bevor sie eingetreten ist. Wir sind die einzigen, die Auskunft geben können über die Beschaffenheit der Angst, die uns befallt, wenn wir an das eigene historische Ende denken. Für uns ist es vorstellbar geworden. Wir wissen, wie es ist, wenn etwas zu Ende geht. Wir sind in der Lage den Schmerz der auslaufenden Art zu beschreiben. Als Beteiligte. Nicht als Beobachter aus der Ferne, für die es eines der vielen Naturspektakel sein könnte, von der die Genesis lebt: Die einen kommen, die anderen gehn, nicht wahr, was solls! Nein, in uns ist der Ubersinn der Selbsterhaltung, die Verrücktheit der Harzer Tanne, die sich im Angsttrieb des Aussterbens mit unnatürlich vielen Zapfen behängt. In uns ist der aufbäumende Widerstand gegen eigene und fremde Gleichgültigkeit. Wir, besselt von dem bleibenden Wunsch zu bleiben, müssen uns um Dinge kümmern, für die andere keine Zeit opfern: um die Sprache in der Familie, im Dorf und Buch, um das Publikum im Theater, um die Busse, die es aus den Dörfern holen und wieder dorthin bringen. Uns kann es wie dem deutschen Theater nicht wurst sein, wieviel Arbeiter und Bauern im Parkett sitzen. Wir spielen fast ausschließlich für sie. Das DT fast ausschließlich für sie nicht. Für uns ist der seit dreißig Jahren nicht renovierte Bühnensaal der Konsumkneipe ein Teil der tausendjährigen ethnischen Tragödie, weil das Amateurspiel und die anderen Spiele der Amateure als die nachfahrigen nationalen Schulen nicht mehr stattfinden können. Ein Laienspektakel am Fuße des Ostroer Burgwalls vermag für einen Abend 5000 Leute herbeizulocken. Wir haben von der Krankheit der Anonymität gehört, aber wir leiden 
nicht unter ihr. Das Vergnügen unserer Vergnügungen ist Fremden oft nicht erklärbar. Die Aufschlüsse der Tagebaue, in deren Gruben unsere Dörfer abfahren, vermögen wir nicht als ökonomischen Erfolg zu begreifen. Wir sehen in ihnen, was sie sind: die einfältigsten der möglichen kreativen Einfalle einer technisch hochentwickelten Nation. Wir besitzen ein drittes Auge. Es ist nur uns gewachsen. Es sieht vieles, wenn nicht alles, anders. Es vermag die Welt zu sehn, wie sie nach der endlichen Abfahrt sein wird. Das Auge der anderen Sicht, der widersprechenden, alternativen. Das Spuren sichernde Auge. Das auf Täter und Töter aus ist. Das Auge des grenzüberschreitenden Weitblicks, des regionalen Weltbürgers, des Mikrowesens, ohne das das angestrebte Makrogemeinwesen eine lächerliche philosophische Utopie bleibt. Wie, wenn der Revierförster seinen Wald zu pflegen vorgibt, dessen Bäume er nacheinander, die seltensten zuerst, absterben läßt. Ohne uns wird kein Wald, kein Staat zu machen sein. Wir sind zu befragen. Mit uns ist zu überprüfen, was geht, was nicht.

Sie sind nicht gefeit vor konservativem Werte-Erhaltungs-Kitsch. Doch es bleibt die große Chance aller talentierten Mandelkrähen. Wir geben die Suche nach der Blauracke auf. Der Mißerfolg, von meinen realistisch denkenden Jungs vorausgesagt, ist $\mathrm{zu}$ ertragen. Wir werden entschädigt von fünfzig oder mehr Jungstörchen, die neben, über, vor uns ihren ersten Weitflug üben, sich sattfressend an Mäusen, die nach dem Grashäcksler nicht schnell genug ihre Löcher finden.

Ein paar Tage später fahre ich noch einmal ins Areal. Mit J., dem Ornithologen. Aber wir unterhalten uns vorsichtshalber über den Storch. Die relativ sichere Majorität, deren Sitten und Gepflogenheiten, ihre schwarz-weiß-rote politische Farbgebung, die große Faszination, die von Großen ausgeht, ihren kräftigen Flügelschlag, den schönen Wechsel ihrer Partnerschaften und die nützliche Großschnäbligkeit. Aber da unterbricht J. meine Ablenkung. Dort! sagt er. Was ist das? Wir springen ins hohe Gras der Wiese. Auf einem Draht der Stromleitung über uns sitzt ein Vogel mit den Maßen des gesuchten. Bevor wir unsere Gläser an die Augen bringen können, läßt er sich fallen und fliegt gaukelnd davon.

Das ist sie! sagt J. Das ist sie!

Ich wills nicht glauben. Widerspreche, zweifle, allerdings in der Hoffnung, daß er recht hat. 